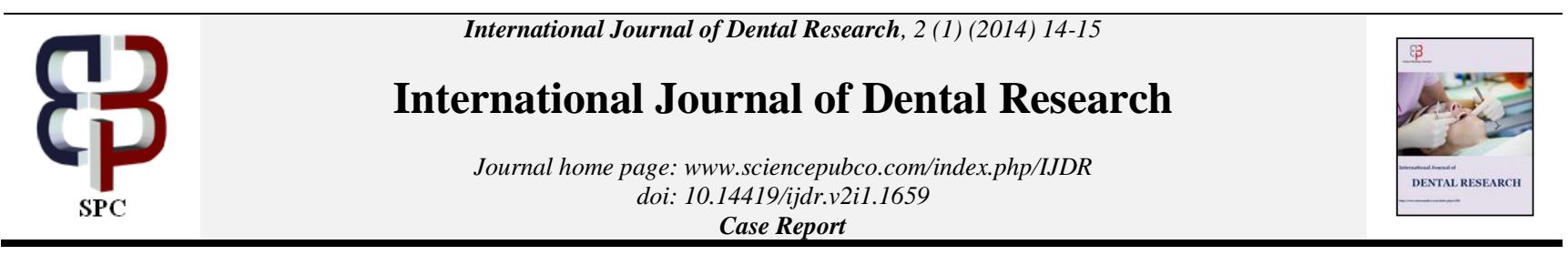

\title{
Root canal treatment in a dilacerated maxillary first molar: A case report
}

\author{
Soheila Darmiani , Maryam Mojtahed Bidabadi* \\ Post Graduate Student of Endodontics, Shahid Beheshti University of Medical Sciences, Tehran, Iran \\ *Corresponding author E-mail: maryam.mojtahed@yahoo.com
}

\begin{abstract}
Aim: The aim of this clinical article is to describe the root canal treatment in dilacerated buccal roots that were detected in a maxillary first molar.

Summary: Success in root canal treatment is achieved after thorough cleaning and shaping followed by the complete obturation of the root canal system. Such treatment may be performed in root canal systems that do not comply with the normal anatomical features described in standard textbooks. The present case describes root canal treatment in dilacerated buccal roots that were detected in a maxillary first molar.
\end{abstract}

Keywords: Dilacerations, root treatment, sever curvature.

\section{Introduction}

A thorough knowledge of root canal morphology is an important aspect of root canal treatment. However, in endodontics, clinicians have to manage teeth with unusual configurations. When a preoperative radiograph reveals an unusual shape, further radiographs should be taken with a different angulation to confirm any unusual anatomical features (Fava \& Dummer 1997).

Most canals have multiple planes of curvature throughout their length (Jafarzadeh \& Abbott 2007). Tomes, in 1848, called such curvatures as "dilacerations" (Tomes 1848). Dilaceration is the result of a developmental anomaly in which there has been an abrupt change in the axial inclination between the crown and the root of a tooth (Hamasha et al.2002 , Ghimire \& Rao 2013) Dilaceration can be seen in both the permanent and primary dentitions. Tooth is considered to have a dilaceration toward the mesial or distal direction if there is a $90^{\circ}$ angle or greater along the axis of the tooth or root. Dilaceration can also be defined as deviation of the apical part of the root by 20 degree or more (Jafarzadeh \& Abbott 2007).

The tapered preparation of the curved canals is the ultimate challenge in endodontics. Meticulous technique will yield a sufficient enlargement of the curved canals. The final result of instrumentation of curved canals may be influenced by several factors such as flexibility and diameter of the endodontic instruments, instrumentation techniques, and the hardness of dentin (Jain \& Tushar 2008). The purpose of this clinical report is to describe theroot canal treatment in dilacerated mesio-buccal and disto-buccal canals that were detected in a maxillary first molar.

\section{Report}

A 24-year-old male patient was referred to the department of endodontics in the dental faculty of ShahidBeheshti University for root canal treatment in his maxillary right first molar.He com plained of pain to cold and hot food and drinks for 10 days. The initial radiograph (Fig.1) disclosed the carious pulp exposure. The medical history was non-contributory. Clinically, the tooth was carious. Periodontal probing revealed a normal intact gingiva. A diagnosis of irreversible pulpitis was confirmed by heat and cold sensitivity tests. The initial radiograph also revealed unusual root morphology of the mesio-buccal and disto-buccal roots which showed a sharp curvature at coronal third. Degree of curvature was determined for buccal canals according to Schneider (Schnieder 1971) technique using parallel radiograph; there was a greater $90^{\circ}$ angle along the axis of the tooth.

After anesthesia with: $1.8 \mathrm{ml}$ of Lidocaine $2 \%$ containing epinephrine 1:80 000 (Persocaine, DarouPakhsh, Iran) and rubber dam isolation access cavity was prepared. A size $10 \mathrm{k}$-file (Dentsply,Maillefer,Ballaigues, Switzerland) was precurved in accordance with the degree of curvature seen in radiograph with mesiobuccal and distobuccal roots. This glide path was ascertained till radiographic working length (WL). Coronal flaring was done by size1, 2, 3 and 4 Gate-glidden drills (Dentsply, Maillefer, Ballaigues, Switzerland) sequentially in a step-back technique. Recapitulation with a size $10 \mathrm{k}$-file was done to check patency and to remove the dentinal debris. WL was then confirmed using an electronic apex locator (Rayapex, VDW, and Germany) in all canals (Fig.2). All the canals were instrumented up to size $15 \mathrm{k}$-file to the WL. Always precurve the instruments to retain the original shape of the canal and prevent transportation. The canals were instrumented passively using HERO 642 (Micro mega,France) rotary files and motor controller device (X-SMART, Dentsply, Maillefer, Ballaigues, Switzerland) according to the manufacture in the following sequence: $20.02,25,02,25.04$, 30,02 . In palatal root, cleaning and shaping was done till 35.04 . The patency of root canals was established by $10 \# \mathrm{~K}$ file. Irrigation during instrumentation was carried out with $2.5 \%$ sodium hypochlorite(Golrang , Iran) by 28-guage needle (Max I-probe, Franklin Park, IL ,USA) and final irrigation with 
17\% EDTA(Calasept, Nordiska Dental, Sweden), which remained for $2 \mathrm{~min}$ in the canal.

After cleaning and shaping, the canals were dried and obturated by the lateral condensation technique using gutta-percha (AriaDent, Iran) and AH26 sealer (DentsplyDeTrey, Germany) (Fig.3). A sterilized cotton pellet was placed in the pulp chamber, the access cavity sealed with Coltosol (AriaDent, Iran) and the patient dismissed. The tooth was restored subsequently by the resident of restorative dentistry and 10 months later the tooth was clinically asymptomatic and radiographically sounds (Fig. 4).

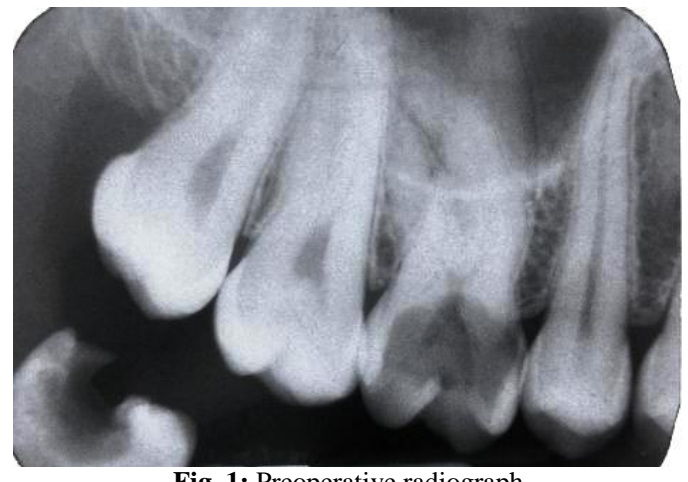

Fig. 1:.Preoperative radiograph

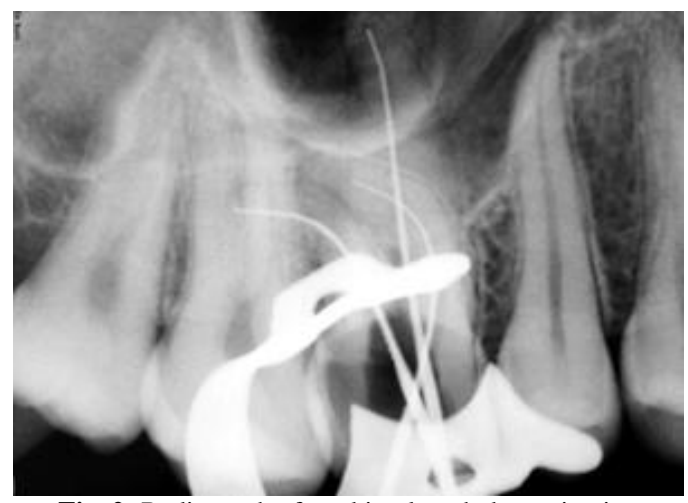

Fig. 2: Radiograph of working length determination

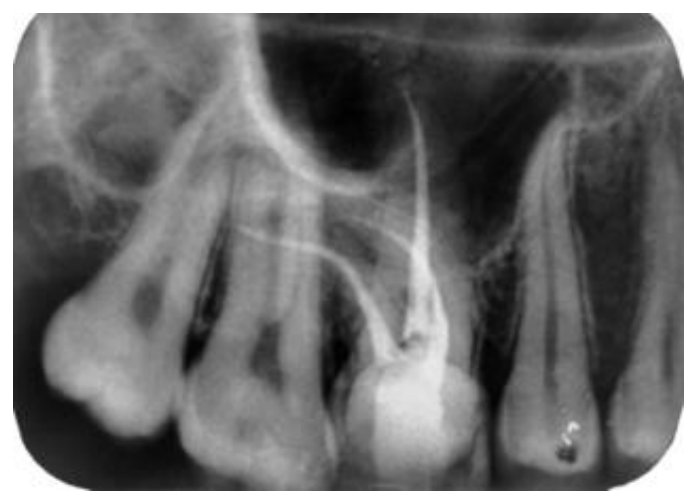

Fig. 3: Radiograph of obturated tooth

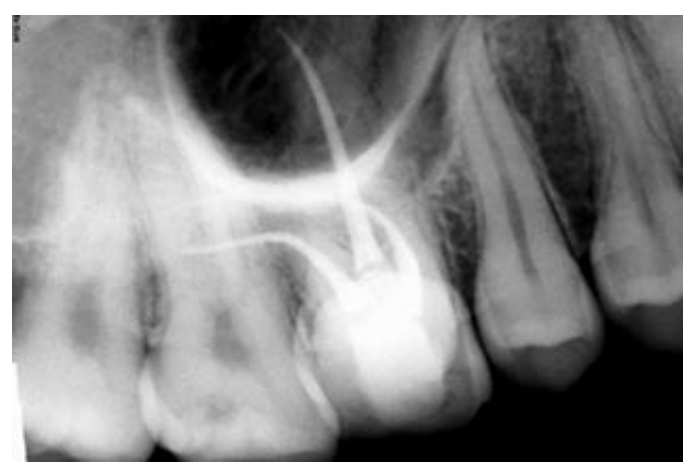

Fig. 4: 10 months later follow up radiograph

\section{Discussion}

One of the most important aspects of endodontic treatment is the cleaning and shaping of the root canals. The most desirable shape of the prepared canal is a progressive taper with the largest diameter at the coronal end and is narrowest at the apical constriction (Schilder 1974, Ansari \& Managing 2012). A progressive taper allows a greater degree of instrument and irrigation contact with the surfaces of the canal walls, thereby enhancing the effectiveness of cleaning (Wiene 2004).

However, the preparation of curved canals especially dilacerated roots presents one of the greatest challenges in endodontics. Failure of root canal treatment in curved canals is mainly due to procedural errors like ledges, perforations, fractured instruments, zip, and canal blockage and elbow creation (Schnieder 1971).

The case presented above were treated with hand instrumentation with K files and NiTi rotary instruments HERO 642 .These NiTi rotary instruments would maintain the original direction of curved root canals, remain canal centering and allow preparation to larger sizes. These flexible instruments produce better shape, using fewer instruments and in a shorter time. Fewer endodontic errors occurred in severe curved canals by using these files.

It is important that a tooth with complex root canal morphology be treated properly the very first time, since endodontic treatment in such teeth is likely to fail if not treated carefully.

From a clinical standpoint, radiographic provide clinicians with the most appropriate method to detect variations in root canal anatomy. Only by correct examination and interpretation of radiographics can the clinician detect such variations and be aware of them before and during endodontic procedures.

\section{Conclusion}

Proper attention should be directed in radiographic assessment to treat dilacerated roots. Current NiTi preparations are a substantial improvement over instrumentation with 0.02 tapered stainless steel files, particularly when used in dilacerated teeth.

\section{Acknowledgement}

The authors deny any conflicts of interest discussed in this manuscript.

\section{References}

[1] Fava LRG, Dummer PMH (1997), Periapical radiographic techniques during diagnosis and treatment. Int Endod J 30, 250-61.

[2] Jafarzadeh H, Abbott PV (2007), Dilaceration: review of an endodontic challenge. J Endod 33, 1025-30

[3] Tomes J (1848), a course of lectures on dental physiology and surgery delivered at the Middlesex Hospital School London: John W. Parker.

[4] Hamasha AA, Al-Khateeb T, Darwazeh A (2002), Prevalence of dilaceration in Jordanian adults. Int Endod J 35, 910-2.

[5] Ghimire N, Rao A. Crown dilaceration. Health Ranaissance 2013; $11(1) ; 86-88$.

[6] Jain N, Tushar S (2008), Curved canals: ancestral files revisited. Indian J Dent Res 19, 267-71.

[7] Schnieder S (1971), Comparison of canal preparations in straight and curved root canals. Oral Surg Oral Med Oral Pathol Oral Radiol Endod 32, 271-5.

[8] Schilder H (1974), Cleaning and shaping of the root canals. Dent Clin N Amer 18, 269-96.

[9] Ansari I, Maria R. Managing (2012), curved canals. Contemp Clin Dent 3(2), 237-241.

[10]Wiene FS (2004), Endodontic therapy. 6th ed. St Louis: Mosby. 